\title{
Bound States of Guided Matter Waves: An Atom and a Charged Wire
}

\section{Citation}

Hau, Lene, Michael Burns, and J. Golovchenko. 1992. Bound states of guided matter waves: An atom and a charged wire. Physical Review A 45(9) (May): 6468-6478.

\section{Published Version}

doi:10.1103/PhysRevA.45.6468

\section{Permanent link}

http://nrs.harvard.edu/urn-3:HUL.InstRepos:11807540

\section{Terms of Use}

This article was downloaded from Harvard University's DASH repository, and is made available under the terms and conditions applicable to Other Posted Material, as set forth at http:// nrs.harvard.edu/urn-3:HUL.InstRepos:dash.current.terms-of-use\#LAA

\section{Share Your Story}

The Harvard community has made this article openly available.

Please share how this access benefits you. Submit a story.

Accessibility 


\title{
Bound states of guided matter waves: An atom and a charged wire
}

\author{
Lene Vestergaard Hau \\ Rowland Institute for Science, 100 Cambridge Parkway, Cambridge, Massachusetts 02142 \\ and Lyman Laboratory, Harvard University, Cambridge, Massachusetts 02138 \\ Michael M. Burns \\ Rowland Institute for Science, 100 Cambridge Parkway, Cambridge, Massachusetts 02142 \\ J. A. Golovchenko \\ Rowland Institute for Science, 100 Cambridge Parkway, Cambridge, Massachusetts 02142 \\ and Lyman Laboratory, Harvard University, Cambridge, Massachusetts 02138
}

(Received 9 May 1991)

\begin{abstract}
We argue that it is possible to bind a neutral atom in stable orbits around a wire charged by a timevarying sinusoidal voltage. Both classical and quantum-mechanical theories for this system are discussed, and a unified approach to the Kapitza picture of effective potentials associated with highfrequency fields is presented. It appears that cavities and waveguides for neutral-atomic-matter waves may be fashioned from these considerations.

PACS number(s): 32.80.Pj, 03.20.+i, 03.65.Ge
\end{abstract}

\section{INTRODUCTION}

The availability of slow atoms from laser cooling sources [1-5] has stimulated us to consider new methods to contain, manipulate, and study these extremely lowenergy neutrals. We are concerned with both classical and quantum-mechanical atomic motions, and we intend to show that the experimental study of both regimes should provide a fertile enterprise that will enable the observation of new phenomena and the development of devices of practical utility.

Because of the possibility of immediately realizing practical structures based on the interaction between a charged wire and neutral polarizable atoms, we shall focus most of our discussion on the resulting attractive $1 / r^{2}$ interaction potential. Nevertheless, a number of our results and methods of analysis are valid for more general systems, and we present them in generic form. In the course of our discussion we comment on the connections with the work of Cook, Shankland, and Wells [6], Combescure [7], and Brown [8], aimed mainly at understanding ion motion in a Paul trap [9], which is governed by the Mathieu equation.

The static $1 / r^{2}$ potential is known mostly to physicists for the role it plays in accounting for the effects of conserved angular momentum in the radial equation of motion for a particle moving in a central field. This effective potential is always repulsive and leads to reduced binding for increasing angular momentum when combined with an attractive force that supports bound states. The attractive $1 / r^{2}$ potential has been discussed classically [10] and quantum mechanically [11-13] and has been of interest mostly for the peculiarities of the motions it engenders rather than for its importance in practical problems. In the following we point out that it is exactly these peculiarities that must and can be over- come to enable the realization of new stable states of matter based on the interaction of polarizable atoms with a charged wire. First we review the basic features of the motion.

The attractive $1 / r^{2}$ potential stands on the border between highly singular and regular potentials for which the centrifugal barrier can keep a particle from passing through the origin. Only motions with angular momentum below a critical value can be bound and all of these do in fact pass through the origin.

Consider now a neutral atom and a line of uniform charge density as might be contained on an extremely thin wire. The atom is polarized by the electric field from the wire and is consequently attracted to the wire by the electric-field gradient. This results in a $1 / r^{2}$ interaction potential when the induced dipole moment is linearly dependent on electric-field strength.

The fate of an atom bound in this way is an unavoidable collision with the wire, which most likely results in absorption of the atom to the surface of the wire or in inelastic reflection. Either of these would severely limit the lifetime of bound states where the atom moves in stable orbits around the wire.

A main motivation of our studies is to show how longlifetime, stable motions can be realized. It is one of our central themes that this can be accomplished by adding a high-frequency component to the static interaction potential. The strategy by which we demonstrate the efficacy of this procedure is remarkably similar to one that can be used for introducing the notion of a centrifugal barrier or effective potential in central force problems. The latter involves a canonical transformation from Cartesian to polar coordinates, which has the favorable consequence of making the angular coordinate ignorable in the transformed Hamiltonian. The radial motion is, however, influenced by an effective potential 
whose magnitude depends on the conserved angular momentum. Similarly, we find that under certain circumstances, a high-frequency potential-energy function can be canonically transformed away, leaving a timeindependent effective potential in its place. The transformed Hamiltonian yields insight into the particle motion, and, in our case, it demonstrates how bound motions of the type we desire can be obtained.

A major achievement of this strategy is that it can be extended from the classical to the quantum regime, which is important for the systems of atomic waveguides and cavities we envision. In this way our approach represents an extension of the analysis presented by $\mathrm{Ka}$ pitza [14] in his study of the inverted pendulum.

\section{BOUND STATES: CLASSICAL DESCRIPTION}

Consider a neutral atom of mass $M$ and polarizability $\alpha$, situated a distance $r$ from the center of a wire with static charge per unit length $q$ and radius $r_{0}$. A grounded cylinder of radius $r_{1} \gg r_{0}$ surrounds the wire to give the system a well-defined capacitance. The atom experiences a radial force of attraction towards the center of the wire, derivable from the potential-energy function

$$
v(r)=-\frac{2 \alpha q^{2}}{r^{2}} \text {. }
$$

A free translational motion along the wire is obtained, and we shall focus on the separate problem of transverse-motion confinement.

The Hamiltonian for the transverse motion of the system is given by

$$
H=\frac{P_{r}^{2}}{2 M}-\frac{2 \alpha q^{2}}{r^{2}}+\frac{L^{2}}{2 M r^{2}},
$$

with radial momentum $P_{r}=M d r / d t$ and conserved angular momentum $L=M r^{2} d \theta / d t$ along the wire. Bound states have energy of transverse motion $E<0$, which can occur only when $L^{2}<4 M \alpha q^{2}$. Integration of the complete equations of motion gives

$$
r=b / \cosh \left[\left(4 M \alpha q^{2} / L^{2}-1\right)^{1 / 2} \theta\right],
$$

with apogee $b$ determined from $v(b)+L^{2} /\left(2 M b^{2}\right)=E$, and

$$
\theta(t)=\frac{\tanh ^{-1}\left[\left(4 M \alpha q^{2} / L^{2}-1\right)^{1 / 2} L t / M b^{2}\right]}{\left(4 M \alpha q^{2} / L^{2}-1\right)^{1 / 2}} .
$$

The maximum time an orbit can have before crashing into the origin is

$$
\Delta T=\frac{2 M b^{2} / L}{\left(4 M \alpha q^{2} / L^{2}-1\right)^{1 / 2}} .
$$

A plot of a characteristic trajectory spiraling into the origin is indicated in Fig. 1. Clearly, maintaining atoms in this system is impossible.

Now imagine adding a time-varying term to the Hamiltonian in Eq. (2) so that it takes the form

$$
H=\frac{P_{r}^{2}}{2 M}-\frac{2 \alpha q^{2}}{r^{2}}+\frac{L^{2}}{2 M r^{2}}+W(r) \cos \omega t .
$$
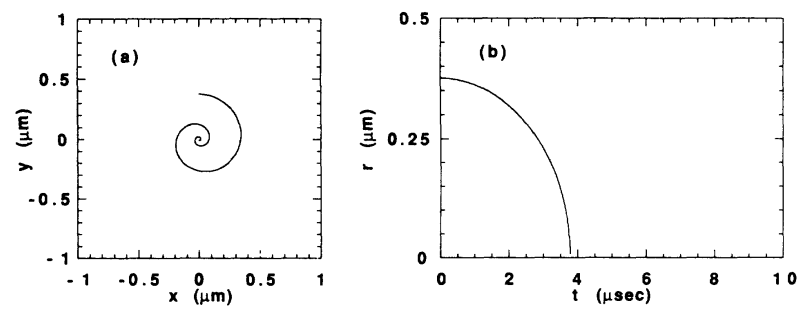

FIG. 1. (a) Orbit for a sodium atom $\left(\alpha=27 \AA^{3}\right)$ with $L=48 \hbar$ attracted to a wire with an applied voltage of $8 / \sqrt{2}$ volts and $r_{0}=0.1 \mu \mathrm{m} . r_{1}=1 \mathrm{~cm}$. (b) Time-dependent radial motion for orbit in (a).

An elementary discussion of the consequences on motion described by such a Hamiltonian is given by Landau and Lifshitz [15] under the condition that $\omega$ is sufficiently high. They show that Newton's equations of motion may then be integrated approximately, yielding a solution for $r$ that consists of a fast component $W^{\prime} \cos \omega t / M \omega^{2}$ superimposed on a slow motion that is governed by the static interaction potential and an effective potential,

$$
V_{\mathrm{Kap}}=\frac{\left(W^{\prime}\right)^{2}}{4 M \omega^{2}},
$$

which we shall refer to as the Kapitza potential. Unfortunately, the aforementioned derivation gives neither a completely clear picture of what the expansion parameter and correction terms to the Kapitza description are, nor a clear path to the role this potential might play in quantum descriptions of the motion. We therefore divert temporarily from our problem of an atom and charged wire to fully develop the Kapitza picture in a classical description.

Consider a time-dependent Hamiltonian system described by

$$
H=\frac{P_{X}^{2}}{2 M}+V(X)+W(X) \cos \omega t
$$

in which $X$ and $P_{X}$ are canonically conjugate variables. To simplify the equations of motion, we want to extract the presumed small, high-frequency component from both the position and momentum by a canonical transformation to a new coordinate $Y$ and momentum $P_{Y}$ related to $X$ and $P_{X}$ by

$$
X \approx Y+\frac{W^{\prime}(Y)}{M \omega^{2}} \cos \omega t
$$

and

$$
P_{X} \approx P_{Y}-\frac{W^{\prime}(Y)}{\omega} \sin \omega t
$$

The displacements in position and momentum on the right-hand sides represent the motion of a free particle exposed to the time-varying potential alone.

The transformations in Eqs. (9) and (10) are not strictly canonical [16], and therefore the motions of $Y$ and $P_{Y}$ are not governed by a Hamiltonian function. Equations (9) 
and (10) are, however, the lowest-order terms for a whole class of transformations that are canonical, where the expansion parameter $\epsilon(Y)$ is given by

$$
\epsilon(Y)=\frac{W^{\prime \prime}(Y)}{M \omega^{2}},
$$

which vanishes in the limit of high frequencies. From these we select one that, though not the simplest possible, corresponds to a particularly simple unitary transformation in the quantum-mechanical description. The next section contains a discussion of the quantum-mechanical case. Remarkably, the detailed canonical transformation for the classical case can be obtained in closed form, but it has little transparent physical content beyond agreeing with Eqs. (9) and (10) to lowest order. We therefore relegate this canonical transformation and its generating function to Appendix A as Eqs. (A4), (A5), and (A2). Our results are greatly simplified by restricting applications to power-law potentials [see Eq. (A1)].

The transformed coordinates satisfy Hamilton's equations of motion generated by a new Hamiltonian. From the theory of canonical transformations [16], which in our case are time dependent, this Hamiltonian is obtained by both augmenting the old one with the time derivative of the generating function [Eq. (A2)] and by expressing the old coordinates in terms of the transformed ones. For the new Hamiltonian, to first order in the expansion parameter $\epsilon(Y)$, we obtain

$$
\begin{aligned}
H_{Y}= & \frac{P_{Y}^{2}}{2 M}+V(Y)+V_{\mathrm{Kap}}(Y) \\
& +V_{\mathrm{Kap}}(Y)\left[4 \frac{V}{W} \cos \omega t-\cos 2 \omega t\right] \\
& +\epsilon(Y)\left[-\frac{P_{Y}^{2}}{M} \cos \omega t\right. \\
& \left.+V_{\mathrm{Kap}}(Y)(\cos \omega t-\cos 3 \omega t)\right]
\end{aligned}
$$

The time-independent part of this Hamiltonian contains the static Kapitza potential in addition to the original $V(Y)$ interaction. The canonical transformation has had the desired effect of reducing the time-varying part of the potential by $\epsilon(Y)$ [note that $V_{\text {Kap }} / W \sim \epsilon(Y)$ ]. In addition, a $P_{Y}^{2}$ term of first order in $\epsilon(Y)$ has appeared. This can be interpreted as a spatially dependent mass correction. The amplitudes of the time-varying parts of the potential, though greatly reduced as compared to the original Hamiltonian (8), still have a spatial modulation of the Kapitza type. Since the ultimate goal is to consider the time-dependent terms as a perturbation, a second canonical transformation is applied to introduce another reduction of these time-varying terms. The generating function and the transformation to the final coordinate $Z$, and momentum $P_{Z}$ are given in Appendix A. The new Hamiltonian $H_{Z}$ is also given there to first order in $\epsilon(Z)$. All lowest-order terms of $H_{Z}$ are indeed time independent. Of course, the utility of this Hamiltonian depends upon neglecting the terms multiplied by $\epsilon(Z)$ which must then be small compared with unity in a sense to be discussed below.

We anticipate that the Hamiltonian $H_{Z}$ generates some motions for which the expansion parameter is small for all their accessible regions of phase space. As a result, the correction terms may be neglected or treated as a perturbation. Under such conditions we identify the Hamiltonian with the Kapitza Hamiltonian

$$
H_{\mathrm{Kap}}=\frac{P_{Z}^{2}}{2 M}+V(Z)+V_{\mathrm{Kap}}(Z),
$$

and, after solving the equations of motion in this representation, the total motion in $X$ space is found by the inverse transformation: $Z \rightarrow Y \rightarrow X$.

We now apply and test the above formalism on the atom-wire problem. To obtain guidance for how small the expansion parameter $\epsilon$ must be for the Kapitza picture to hold, we compare with computer calculations where trajectories are obtained from the original Hamiltonian (8). For the charge on the wire, we shall assume a purely sinusoidal time variation at frequency $\omega / 2$ and amplitude $Q$, which gives

$$
q^{2}=Q^{2} \frac{1-\cos \omega t}{2} .
$$

Thereby, the atom-wire Hamiltonian assumes the form

$$
H=\frac{P_{r}^{2}}{2 M}+\frac{1}{r^{2}}\left(\frac{L^{2}}{2 M}-\alpha Q^{2}\right)+\frac{\alpha Q^{2}}{r^{2}} \cos \omega t .
$$

In the Kapitza limit, taking $r=X \rightarrow Y \rightarrow Z$, the motion is governed by a time-independent Hamiltonian,

$$
H_{\mathrm{Kap}}=\frac{P_{Z}^{2}}{2 M}+\frac{1}{Z^{2}}\left(\frac{L^{2}}{2 M}-\alpha Q^{2}\right)+\frac{1}{Z^{6}} \frac{\alpha^{2} Q^{4}}{M \omega^{2}},
$$

which gives rise to a new conserved quantity $E_{\mathrm{Kap}}=H_{\mathrm{Kap}}$. The total potential in $H_{\mathrm{Kap}}$ has a minimum $U_{\min }$ at $Z_{\min }$, and if we convert the coordinate $Z$ and energy $E_{\mathrm{Kap}}$ into reduced variables $\zeta$ and $\delta$, we may write (16) as

$$
\frac{P_{Z}^{2}}{2 M}+U_{\min }\left(\frac{3}{2 \xi^{2}}-\frac{1}{2 \xi^{6}}\right)=\delta\left|U_{\min }\right|,
$$

with

$$
\zeta=\frac{Z}{Z_{\min }}, \quad Z_{\min }^{4}=\frac{\alpha^{2} Q^{4}}{M \omega^{2}} \frac{3}{\alpha Q^{2}-\frac{L^{2}}{2 M}},
$$

and

$$
\delta=E_{\mathrm{Kap}} /\left|U_{\min }\right|, \quad U_{\min }=-\frac{2}{Z_{\min }^{6}} \frac{\alpha^{2} Q^{4}}{M \omega^{2}} .
$$

The potential-energy terms of (16) are plotted in Fig. 2. They consist of a $1 / Z^{2}$ interaction (just the timeindependent potential in the original Hamiltonian) and a repulsive $1 / Z^{6} \mathrm{Kapitza}$ term. Clearly, for appropriate initial conditions on the motion, bound states are suggested, which are constrained to occupy regions away from 


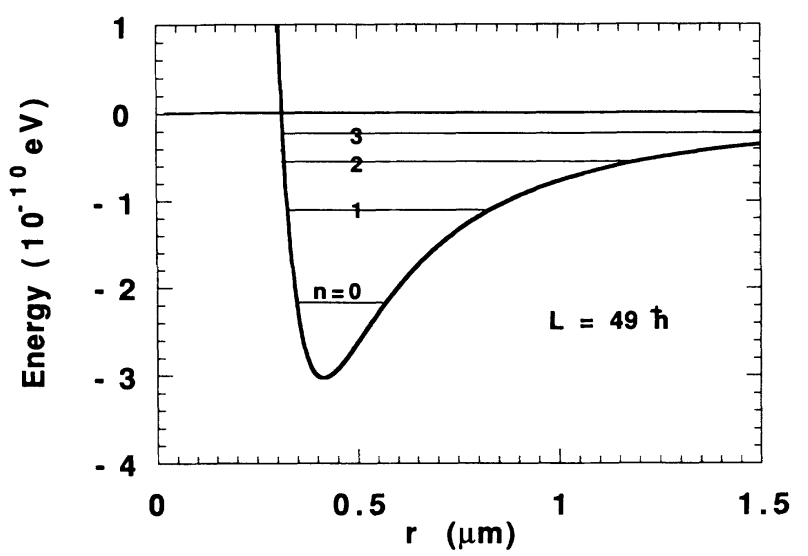

FIG. 2. Total effective potential in the Kapitza picture for a sodium atom, $L=49 \hbar, r_{0}=0.1 \mu \mathrm{m}, r_{1}=1 \mathrm{~cm}$, and an applied peak voltage of $8 \mathrm{~V}$ at $\omega / 2=2 \pi \times(400 \mathrm{kHz})$. Shown also are the lowest quantized energy levels for this system.

the origin or wire in our problem.

Figures 3(a) and 3(b) show plots of a stable bound trajectory obtained from a numerical integration of the original dynamical equations with initial conditions chosen to correspond to a bound motion in the Kapitza picture. The separation into fast and slow motion, inherent in our canonical transformations, is clearly seen. However, as can be seen in Figs. 3(c) and 3(d), even for initial conditions that suggest bound states of the Kapitza Hamiltonian, the actual motion may not be stable. The difference between these two cases is related to the value of the expansion parameter $\boldsymbol{\epsilon}(\boldsymbol{Z})$.

We empirically find that stable motion is obtained for a
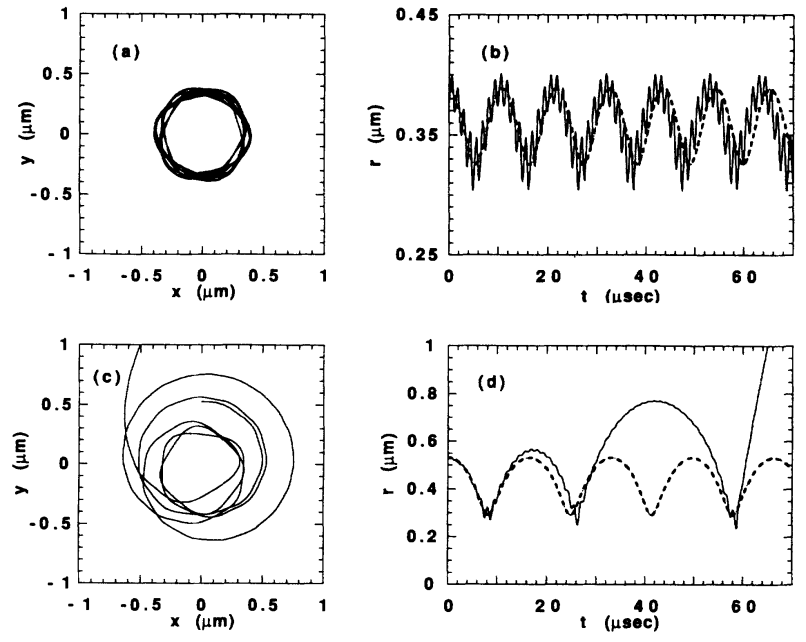

FIG. 3. (a) Bound orbit with conditions as in Fig. 1 but with an applied sinusoidal voltage of $8 \mathrm{~V}$ peak and $\omega / 2=2 \pi \times(400$ $\mathrm{kHz}$ ). $\epsilon=0.20, \delta<0$. (b) Stable time-dependent radial motion in (a) shown as a solid line. The dashed line shows Kapitza motion for $Z$. (c) Unstable orbit with $\epsilon=0.32, \delta<0$. (d) Unstable time-dependent radial motion in (c) shown as a solid line. The dashed line shows Kapitza motion for $Z$. wide range of initial conditions as long as $\epsilon(Z)$ is less than a critical value at the inner turning point in the potential of Fig. 2. The motion predicted by the Kapitza Hamiltonian is then a good approximation to the slowly varying part of the trajectory. We find that bound states of extremely long lifetime compared to that in Eq. (5) will be obtained when

$$
\epsilon=\epsilon\left(Z_{\text {in }}\right)<0.28
$$

at the distance of closest approach to the wire, $Z_{\text {in }}$. This distance is obtained from Eq. (17) with $P_{Z}=0$ and gives, when inserted in (11), the $\delta$-dependent lower limit on $L^{2}$ in (20a),

$$
2 M \alpha Q^{2}[1-0.28 f(\delta)]<L^{2}<2 M \alpha Q^{2},
$$

where

$$
f(\delta)=\frac{1}{2} \frac{\left[\cos \left[\frac{1}{3} \cos ^{-1}\left(1-2 \delta^{2}\right)+\frac{4 \pi}{3}\right]+\frac{1}{2}\right]^{2}}{\delta^{2}}
$$

The upper limit $\left(L_{\max }^{2}\right)$ for $L^{2}$ in Eq. (20a) is simply determined from the requirement that the total radial effective potential energy be negative. Of course, the reduced energy $\delta$ must be negative for bound states, i.e., $-1 \leq \delta<0$. Note the dependence of the left-hand side of (20a) on $\delta$. In Fig. $4, L_{\min }^{2}$ is given as the left-hand side of (20a) with $\delta=-1$.

Figure 4 shows regions of $E_{\mathrm{Kap}}-L$ space where the Kapitza picture should be valid. The stability region is delimited by the obvious constraints that $U_{\text {min }}<E_{\text {Kap }}<0$, and by the upper and lower $L$ values discussed in connection with Eq. (20). The bound motion shown in Figs. 3(a) and 3(b) has $\epsilon=0.20$ and occurs at point $A$ in Fig. 4, which lies within the stability region. Small deviations from the Kapitza motion, such as those

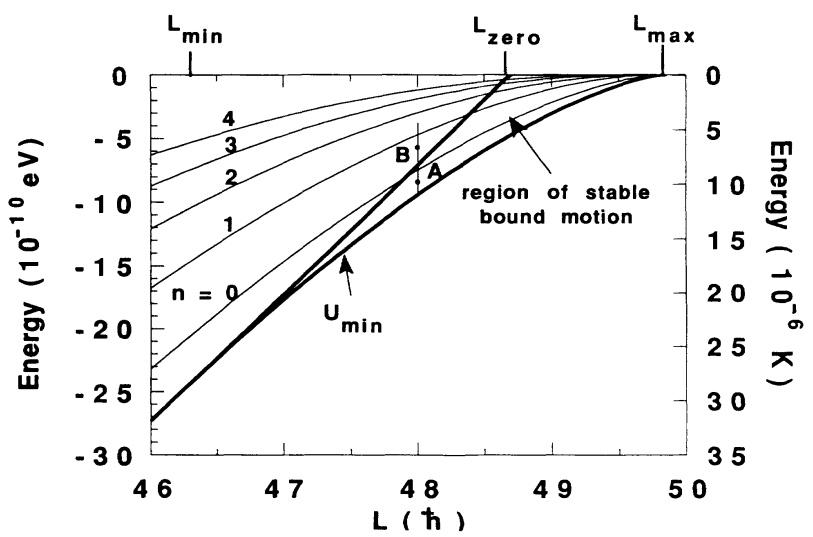

FIG. 4. Regions of stable bound motion for sodium atoms, with sinusoidal voltage of $8 \mathrm{~V}$ peak, $\omega / 2=2 \pi \times(400 \mathrm{kHz})$. The upper boundary connecting $L_{\min }$ with $L_{\text {zero }}$ is obtained from the left-hand side of condition (20a). $L_{\max }$ comes from the righthand side of (20a). Points $A$ and $B$ correspond to the stable and unstable trajectories in Fig. 3. WKB energy levels from Eq. (B7) are also indicated. 
seen in Fig. 3(b), can presumably be accounted for by higher-order terms in the Hamiltonian [see (A10)]. Point $B$ corresponds to the motion of Figs. 3(c) and 3(d), with $\epsilon=0.32$, which is unstable and ultimately escapes to infinity. At the distance of closest approach to the wire, it is condition (19) that is violated, so the left-hand side of (20a) is not fulfilled for this trajectory. This leads to a breakdown of the Kapitza picture. For trajectories like $B$ that reemerge into regions where $\epsilon(Z)<0.28$, motion will again be governed by the Kapitza Hamiltonian but with completely new values of $\delta$ that are very sensitive to the original initial conditions of the problem.

A complementary and more global view of the system dynamics is provided by the Poincare plots shown in Fig. 5. The motion in two-dimensional radial phase space is sampled at the frequency of the ac drive. Keeping the amplitude and frequency of the sinusoidal voltage fixed, we varied the initial position and velocity for constant angular momentum to obtain trajectories for the range of energies $E_{\mathrm{Kap}}$ shown in Fig. 4 by the vertical line (at $L=48 \hbar$ ) crossing the boundary of the stability region. For the largest binding energies (corresponding to the smallest values of $\epsilon$ ), the slow motion is essentially governed by the Kapitza Hamiltonian, which gives rise to the concentric rings (cross sections of tori) in Fig. 5. As this energy is decreased, the lengthening period of the slow motion comes into resonance with an integral multiple of the period for the ac drive, and the nonlinearities of the problem stabilize that motion, forming the ring of nine islands in Fig. 5 (corresponding to $\epsilon=0.23$ ). This is a subtle indication of the imminent breakdown of the $\mathrm{Ka}$ pitza picture.

Further decreases in the binding energy (and increases in $\epsilon$ ) give successively more complicated overlapping resonances (and lack of energy conservation expected of the Kapitza picture). We conjecture that the stable and bound motions in this phase space correspond to those in time-independent nonlinear problems where the

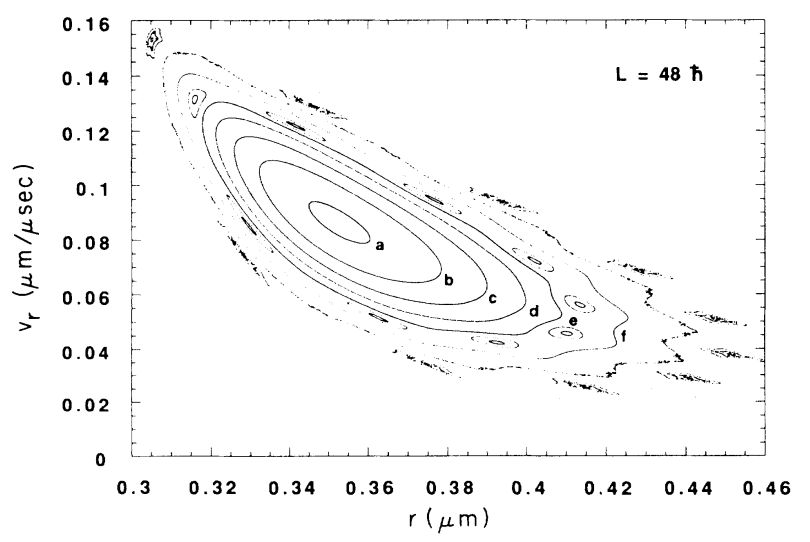

FIG. 5. Poincare plots of trajectories for $L=48 \hbar$ along the vertical solid line of Fig. 4 with energies from $U_{\min }$ to just beyond the line of stability. $a,-8.9 \times 10^{-10} \mathrm{eV}, \epsilon=0.16$; $b,-8.7 \times 10^{-10} \mathrm{eV}, \epsilon=0.19 ; c,-8.5 \times 10^{-10} \mathrm{eV}, \epsilon=0.20$; $d,-8.3 \times 10^{-10} \mathrm{eV}, \epsilon=0.21 ; e,-8.1 \times 10^{-10} \mathrm{eV}, \epsilon=0.23$; $f,-7.8 \times 10^{-10} \mathrm{eV}, \epsilon=0.24$.
Kolmogorov-Arnold-Moser theorem [17,18] has been applied and has provided guidance on the existence and number of such orbits.

\section{BOUND STATES: QUANTUM DESCRIPTION}

We begin immediately by considering the possibility of a Kapitza description under conditions where a quantum-mechanical description is called for. In particular, we note that de Broglie wave lengths for the atoms can indeed be comparable with orbital dimensions at the low energies we are discussing.

Thus we return to our original time-dependent Hamiltonian,

$$
H_{X}=\frac{P_{X}^{2}}{2 M}+V(X)+W(X) \cos \omega t .
$$

The dynamical variables are now interpreted as operators in the Heisenberg picture, which offers the closest correspondence between classical and quantummechanical descriptions. In quantum mechanics, canonical transformations are represented by unitary transformations, and accordingly we anticipate that a unitary transformation that accounts for the fast motion will simplify the description under high-frequency conditions and again lead us to the Kapitza Hamiltonian.

The generating function in (A2), which led to the classical canonical transformations, corresponds to the quantum-mechanical unitary operator

$$
\begin{aligned}
U= & \exp \left[-\frac{i}{\hbar} \int \frac{Y}{\hbar} d y \frac{W^{\prime}(y)}{T^{2}(y)} \frac{\sin \omega t}{\omega}\right] \\
& \times \exp \left[-\frac{i}{2 \hbar}\left[P_{Y} \frac{W^{\prime}(Y)}{M \omega^{2}}+\frac{W^{\prime}(Y)}{M \omega^{2}} P_{Y}\right] \cos \omega t\right],
\end{aligned}
$$

where the function $T$ is defined in (A3). The first factor is a gauge transformation, which accounts for the proper momentum displacement, and the last factor results in the translation in position. The transformation relations for coordinate and momentum operators are obtained from

$$
\begin{aligned}
& X=U^{\dagger} Y U, \\
& P_{X}=U^{\dagger} P_{Y} U,
\end{aligned}
$$

and the result is given in Appendix B. The classical relations [Eqs. (A4) and (A5)] are regained apart from the symmetrization needed to insure that the momentum operator is Hermitian. The Hamiltonian, which generates the equations of motion for these new operators in the form

$$
\begin{aligned}
& i \hbar \frac{d Y}{d t}=\left[Y, H_{Y}\right], \\
& i \hbar \frac{d P_{Y}}{d t}=\left[P_{Y}, H_{Y}\right],
\end{aligned}
$$

is given by 


$$
\begin{aligned}
H_{Y}= & H_{X}+i \hbar U \frac{\partial U^{\dagger}}{\partial t} \\
= & \frac{P_{Y}^{2}}{2 M}+V(Y)+V_{\mathrm{Kap}}(Y)+V_{\mathrm{Kap}}(Y)\left[4 \frac{V}{W} \cos \omega t-\cos 2 \omega t\right] \\
& -\frac{1}{4 M}\left[P_{Y}^{2} \epsilon(Y)+2 P_{Y} \epsilon(Y) P_{Y}+\epsilon(Y) P_{Y}^{2}\right] \cos \omega t+\epsilon(Y) V_{\mathrm{Kap}}(Y)(\cos \omega t-\cos 3 \omega t)+\cdots
\end{aligned}
$$

This result agrees with the classical one in Eq. (12) except for the mass-correction term, which now appears in symmetrized form due to the noncommuting behavior of $P_{Y}$ and $Y$. Note again the appearance of the Kapitza potential and the expansion parameter $\epsilon(Y)$ in this operator equation.

As in the classical case, a second transformation, to coordinate operator $Z$ and momentum $P_{Z}$, is required to eliminate the time-dependent Kapitza-like terms. We give the corresponding unitary operator and the associated Hamiltonian $H_{Z}$ in Appendix B. Since this second transformation does not alter the mass-correction term to first order in $\epsilon(Z)$, the Hamiltonian differs from the classical result only by the symmetrization of the masscorrection term as was found in $H_{Y}$. To lowest order, we obtain the Kapitza Hamiltonian operator

$$
H_{\mathrm{Kap}}=\frac{P_{Z}^{2}}{2 M}+V(Z)+V_{\mathrm{Kap}}(Z) .
$$

Let the system be in state $|\Psi\rangle$, and consider the probability amplitude for the observable corresponding to the coordinate operator $Z$ to have the value $z$ at time $t$. We denote it by $\Psi(z, t)$. From the equations of motion (24) for the operators, we find that the amplitude $\Psi(z, t)$ must solve the Schrödinger equation

$$
\begin{aligned}
i \hbar \frac{\partial}{\partial t} \Psi(z, t)= & \left.-\frac{\hbar^{2}}{2 M} \frac{\partial^{2}}{\partial z^{2}}+V(z)+V_{\mathrm{Kap}}(z)\right] \Psi(z, t) \\
& +\Delta H_{Z}\left(z,-i \hbar \frac{\partial}{\partial z}, t\right) \Psi(z, t)
\end{aligned}
$$

Here $\Delta H_{Z}$ [see (B4)] consists of the higher-order terms in $\epsilon$. We have replaced the coordinate and momentum operators $Z$ and $P_{Z}$ by their representatives in the coordinate representation. Due to the periodicity in time of $\Delta H_{Z}$, we may choose solutions $\Psi(z, t)$ as Bloch functions.

The classical coordinate transformations (A4) and (A8) relate eigenvalues for the $X, Y$, and $Z$ operators. Hence we obtain the wave function in the $X$ representation from the solution $\Psi(z, t)$ in the $Z$ representation by using the inverse of these transformations,

$$
\Psi(x, t)=\Psi(z(y(x)), t)\left[\frac{\partial z}{\partial y} \frac{\partial y}{\partial x}\right]^{1 / 2} .
$$

The Jacobians originate from the normalization of the coordinate operator eigenstates. Since the canonical transformations are periodic in time, the Bloch-function character of the solution is preserved.

We anticipate that for small values of the "expansion parameter," states in the $Z$ representation develop according to the Kapitza Hamiltonian. Solutions of Eq. (27) may then be chosen as stationary states of this operator, i.e.,

$$
\Psi_{E}(z, t)=\exp \left(-i E_{\mathrm{Kap}} t / \hbar\right) \Psi_{E}(z, 0),
$$

where $\Psi_{E}$ is an eigenfunction for the Kapitza Hamiltonian corresponding to energy $E_{\mathrm{Kap}}$. Transformation back to the $X$ representation causes a translation and a rescaling of the argument of the wave functions, which make explicit the high-frequency effects hidden by our transformations to the Kapitza picture.

Corrections to the Kapitza approximation are obtained through perturbation theory as long as the appropriate matrix elements involving the "expansion parameter" are small. Compared to the classical case, there is, of course, the problem that the "expansion parameter" is now an operator. We are ultimately interested in the stability of states, and, for our atom-wire problem, we shall give estimates for the lifetime of eigenstates of the Kapitza Hamiltonian, based upon the golden rule.

In addition to the fact that the above quantum description corresponds to the apparently successful classical description, note that for quadratic power potentials it yields the exact solution to the problem of the Paul trap for ions in the high-frequency limit. There our results reduce to those of Combescure [7] and Brown [8], who have taken advantage of the linear (Mathieu) equations of motion for the Paul trap to find exact solutions at all frequencies. Our case is nonlinear, and the methods we have developed yield useful results only at high frequencies. A transformation corresponding to a part of (22) was performed by Cook, Shankland, and Wells [6]. This yields a translation in the momentum operator only and results in an incomplete description of the Kapitza picture.

We now return to the neutral-atom-wire problem. The time-dependent Schrödinger equation for the transverse motion is given by

$$
\begin{aligned}
i \hbar \frac{\partial}{\partial t} \Psi(r, \theta, t)= & {\left[\frac{-\hbar^{2}\left[\frac{1}{r} \frac{\partial}{\partial r} r \frac{\partial}{\partial r}\right]}{2 M}+\frac{1}{r^{2}}\left[\frac{L^{2}}{2 M}-\alpha Q^{2}\right]\right.} \\
& \left.+\frac{\alpha Q^{2}}{r^{2}} \cos \omega t\right] \Psi(r, \theta, t)
\end{aligned}
$$

Since the angular momentum operator $L$ involves the an- 
gle only, we can separate the variables and choose states of the form

$$
\Psi(r, \theta, t)=R(r, t) \frac{e^{i l \theta}}{\sqrt{2 \pi}} \quad l=0, \pm 1, \pm 2, \ldots,
$$

which are eigenstates for the $L$ operator with eigenvalues $l \hbar$. The radial equation may be written in terms of $\chi(r, t) \equiv R(r, t) \cdot \sqrt{r}$ as

$$
\begin{aligned}
i \hbar \frac{\partial}{\partial t} \chi(r, t)= & -\frac{\hbar^{2}}{2 M} \frac{\partial^{2}}{\partial r^{2}}+\frac{1}{r^{2}}\left[\hbar^{2} \frac{l^{2}-\frac{1}{4}}{2 M}-\alpha Q^{2}\right] \\
& \left.+\frac{\alpha Q^{2}}{r^{2}} \cos \omega t\right] \chi(r, t)
\end{aligned}
$$

which is a one-dimensional time-dependent Schrödinger equation corresponding to a Hamiltonian of the form (21) with

$$
V(r)=\frac{\frac{\hbar^{2}\left(l^{2}-\frac{1}{4}\right)}{2 M}-\alpha Q^{2}}{r^{2}}, W(r)=\frac{\alpha Q^{2}}{r^{2}} .
$$

(The $\hbar^{2} / 4$ term above originates in the two-dimensional nature of the problem.)

By letting the operator $r=X \rightarrow Y \rightarrow Z$, we obtain a stationary equation in the quantum-mechanical Kapitza picture, which in reduced variables [see Eq. (18) with $\left.L^{2} \rightarrow \hbar^{2}\left(l^{2}-\frac{1}{4}\right)\right]$ is

$$
-\frac{d^{2} \chi}{d \xi^{2}}+u_{\min }\left(\frac{3}{2 \xi^{2}}-\frac{1}{2 \xi^{6}}\right) \chi=\delta\left|u_{\min }\right| \chi,
$$

where

$$
u_{\min }=U_{\min } / \frac{\hbar^{2}}{2 M Z_{\min }^{2}} .
$$

Although it is possible to integrate this equation by numerical means, we have found that in the phase-space regions of interest here $\left(u_{\min }>10\right)$, the Wentzel-KramersBrillouin (WKB) approximation yields very accurate results for the reduced bound state energies in an analytic form. In the limit of weak binding $(\delta \rightarrow 0)$ they are given as

$$
\begin{aligned}
& \delta=-C \exp \left[-\left(n+\frac{1}{2}\right) \pi\left(\frac{8}{3\left|u_{\min }\right|}\right]^{1 / 2}\right], \\
& C=\frac{12 \sqrt{3}}{\exp (3)} .
\end{aligned}
$$

This approximation can actually be used in the range of $|\delta|<0.9$, where the error introduced for large binding is only a few percent. The eigenvalues are shown graphically in Figs. 2 and 4, where they are superimposed on the classical stability region. In Appendix B, results from the numerical integration are compared to WKB energies.

Having found energy eigenvalues and corresponding eigenstates for the Kapitza Hamiltonian of the atom-wire problem, we can now return to the question of stability of these states. A conservative estimate of the transition rate between states $\chi_{i}$ and $\chi_{f}$ is given by the golden rule,

$$
\begin{aligned}
\Gamma=\frac{\pi}{2 \hbar} \sum_{n=1,2,3, \ldots} & \left|\left\langle\chi_{f}|\Delta H(n \omega)| \chi_{i}\right\rangle\right|^{2} \\
& \times \frac{d N}{d E}\left(E_{\mathrm{Kap}}^{f}=E_{\mathrm{Kap}}^{i} \pm n \hbar \omega\right),
\end{aligned}
$$

where $d N / d E$ is the density of Kapitza states, and the "Fourier components" $\Delta H(n \omega)$ of the perturbation are obtained from (B4) as the amplitudes of the $\cos (n \omega t)$ terms.

We shall consider the lifetime of the ground state in Fig. 2 that is deeply inside the quantum regime. The energy quanta of the radio-frequency driving terms in the perturbation are much larger than the depth of the effective potential in the Kapitza picture. The perturbation thus couples the ground state to free states. With the atom-wire potentials inserted into Eq. (B4), where we include only the $\cos (\omega t)$ terms, we obtain from (37)

$$
\Gamma=\omega \frac{9}{16 \sqrt{\delta_{f}}} \frac{u_{\min }^{4}}{l_{\max }^{6}}|I|^{2},
$$

with

$$
\begin{gathered}
I=\int d \zeta\left[\chi_{f}^{*} \chi_{i}\left[\frac{33}{10 \xi^{10}}-\frac{6}{\zeta^{6}}-\frac{4 \delta_{i}+\left(6 l_{\max }^{2} /\left|u_{\min }\right|^{3 / 2}\right)}{\zeta^{4}}\right]\right. \\
\left.+\frac{d \chi_{f}^{*}}{d \zeta} \chi_{i} \frac{8}{u_{\min }} \frac{1}{\zeta^{5}}\right] .
\end{gathered}
$$

Here $\delta_{f}$ is the energy of the final states in reduced units, and $\hbar l_{\max }=\left(2 M \alpha Q^{2}\right)^{1 / 2}$ is the maximum $L$ value given by the right-hand side of (20a).

The result is a ground-state lifetime of $3 \mathrm{msec}$. This is much shorter than the lifetime predicted by the classical description, where we found stability over seconds. The reason for this difference is clear: the ground state tunnels into classically forbidden regions where the expansion parameter is large. We can approach the classical regime by choosing larger values for the voltage, frequen$c y$, and angular momentum. It is then possible to obtain stability of the states for seconds or more. For these cases, the energy of the radio-frequency quanta becomes much smaller than the depth of the potential, and, accordingly, scattering between Kapitza states does not necessarily lead to loss of the atom. Although the quantum-mechanical lifetime found is much smaller than the classical result, we still see enormous enhancements when compared to the static case of Fig. 1.

\section{DISCUSSION}

The bulk of the preceding has dealt with establishing and evaluating the applicability of the Kapitza picture to the atom-wire and related problems. All of our classical and quantum-mechanical intuitions about the atomic states are deduced from this description. Even where the exact equations of motion were directly integrated to study stability, the interesting parameters and initial conditions were obtained from the Kapitza picture. Note that expansions or linearization of the equations of motion around exact solutions were not necessary. In ad- 
dition, the mathematical formulation of the Kapitza picture for power-law potentials led naturally to an expansion parameter that serves as a useful guide to the phasespace regions where the approximation is useful.

Here we shall give a physical argument explaining why $W^{\prime \prime} / M \omega^{2}$ is an appropriate parameter controlling the applicability of the Kapitza picture to our problem. Clearly, the description breaks down when the "local" frequency for the slow motion becomes comparable to the high frequency of the time-dependent interaction, since then energy transfers to the slow motion can be large and unpredictable. The local frequency is proportional to the square root of the curvature of the total potential in the Kapitza picture. The strictest requirement is obtained at the inner turning point, where the potential is approximately the Kapitza potential only,

$$
1>\frac{1}{\omega}\left(\frac{V_{\mathrm{Kap}}^{\prime \prime}}{M}\right)^{1 / 2} \approx \frac{W^{\prime \prime}}{M \omega^{2}},
$$

which is exactly our expansion parameter.

It should be emphasized that this requirement for the local frequency is a stronger constraint than the requirement that the overall oscillation frequency for the slow motion be small compared to the driving frequency. This global oscillation frequency for the atom-wire problem is determined mainly by the long-range, attractive $1 / r^{2}$ potential. In the case of the Paul trap, however, the global and local oscillation frequencies coincide.

We see that for the Kapitza picture to be valid, the local frequency of the slow motion should be small compared to the drive frequency at any point in the oscillation. For our problem we then conclude that although the global frequency becomes smaller for weaker binding, the inner turning point moves closer to the origin, where the local frequency is larger. The fact that instability occurs at smaller rather than larger binding energies in Fig. 4 is thus explained.

The theoretical discussion in the preceding sections clearly shows that enormous enhancements can be achieved in the time during which a low-energy neutral atom may occupy stable, bound orbits around a wire. Realization of such a system will most likely rely on the combined technology of laser cooling atoms to the microand nano-electron-volt regions and the fabrication of structures like small wires of accurate dimensions on micro- and submicrometer length scales. The plots we presented above were all for what we believe to be realistic experimental conditions, none of which is beyond current capabilities. We expect the observation of these states to occur shortly, followed by more-detailed spectroscopy and experimental probes of the atomic motions.

Since our atoms are uncharged, the coupling to external electromagnetic waves at frequencies comparable to the slow atomic motions is quite small. Indeed, the radiative lifetime of the bound states need hardly be considered. The system should surely be probed spectroscopically through signals applied directly to the wire.

Although we have for simplicity discussed only the application of a sinusoidal voltage to the wire, we have also studied the case where an additional constant voltage is applied. The methods of this paper can be applied equally well to this case, yielding similar bound-state motions. The additional parameter adds the possibility of modifying these states adiabatically during an experiment.

It is quite interesting to consider the possible mechanisms that will limit the lifetime of the bound states. First we must admit that in spite of all our preceding theoretical and computer studies, we do not have a proof of the possibility of absolute binding for infinitely long periods of time for any classical or quantum-mechanical motions that we have discussed. It is a conjecture that the terms in the Hamiltonian beyond those of the Kapitza type result in secular phase perturbations only. Our intuition that this is true in the classical limit is firmly based upon computer simulations following the bound system over millions of cycles.

Even if the classical trajectories could be shown to be absolutely stable, it is clear that this will not be true for the quantum-mechanical motion. Tunneling to classically forbidden regions, where the expansion parameter is large, can lead to transitions between Kapitza states and ultimate loss of binding. The effect of tunneling to these regions, or to the surface of the wire, can be probed experimentally. There is an additional quantummechanical effect that we have not discussed that may also play a role in the lifetime of stable states: in our quantum treatment the charge on the wire was taken to be a classical, time-dependent parameter. This is, of course, an approximation. It must ultimately be considered as a quantum-mechanical dynamical variable in its own right, and this can affect the stability of the atomic states.

We are intrigued by the fact that the atom-wire system may be investigated over a range of conditions spanning classical to quantum-mechanical regimes. The existence or interpretation of chaos in quantum systems is a topic of current interest, and we expect the experimental and theoretical study of the boundaries to motion governed by the Kapitza picture to contribute to our insight here.

However, in the final analysis, we believe that the ultimate utility of the new system we propose will depend directly on the lifetime of those orbits we imagine to be the most stable. Under favorable conditions, atomic constants and interactions between surfaces and atoms may be determined. Reference systems that depend on the values of such constants could be established. Transport of atoms in well-defined states over large distances or containment over long times would be achieved. The possibility of large numbers of atoms being contained simultaneously must also be considered. In this connection it is extremely important to note that internal heating from interparticle interactions will be strongly suppressed for neutral-particle confinement compared to that in ion traps. The distinction between Bose and Fermi particles must also be taken into account, and achievement of a bound, condensed state in the former case is an exciting possibility. Confinement along the wire's length is then required as well, and we anticipate that nonuniformities in the wire or modification of external-fieldforming surfaces will satisfy this requirement.

The general utility of the Kapitza picture in under- 
standing and unifying a number of diverse physical phenomena perhaps also deserves some note. Strong focusing in accelerators, channeling of charged particles in crystal lattices, trapping of ions in the Paul trap, and the Lamb shift of atomic physics as well as the new atomic states we have introduced here may all be viewed as resulting from the peculiar rectification of high-frequency driving forces in dynamical equations of motion. We hope our work will serve to promote interest in and understanding of this point of view.

\section{ACKNOWLEDGMENTS}

We would like to acknowledge stimulating discussions and advice from our many colleagues and friends at the Rowland Institute and Harvard and Århus Universities. These include Professor P. Martin, Professor E. Purcell, Professor J. Lindhard, Professor E. Eilertsen, and in particular Professor D. Hershbach, who pointed out the elliptic-function analytic solution to the WKB problem. One of us (L.V.H.) greatly appreciates the support provided by the Carlsberg Foundation.

\section{APPENDIX A: CLASSICAL TREATMENT}

Here we shall give the explicit expressions for the canonical transformations that lead to the Kapitza picture. We restrict ourselves to power potentials of the form

$$
\begin{aligned}
& V(X) \propto X^{j}, \\
& W(X) \propto X^{j} .
\end{aligned}
$$

The generating function for the first transformation of the classical treatment is

$F\left(P_{X}, Y\right)=-P_{X} Y S(Y)-\int^{Y} d y \frac{W^{\prime}(y)}{T^{2}(y)} \frac{\sin \omega t}{\omega}$

where the scaling functions $S$ and $T$ for coordinate and momentum, respectively, are given by

$$
\begin{aligned}
& S(Y)=\left[1-\frac{(j-2)}{(j-1)} \epsilon(Y) \cos \omega t\right]^{1 /(2-j)}, \\
& T(Y)=\left[1-\frac{(j-2)}{(j-1)} \epsilon(Y) \cos \omega t\right]^{(j-1) /(j-2)} .
\end{aligned}
$$

Here the symbol $j$ denotes the power of the interaction potentials. For the atom-wire problem $j=-2$, and for the Paul trap $j=2$. The generating function in (A2) results in the following relations between new and original coordinates and momenta, $Y, P_{Y}$ and $X, P_{X}$, respectively,

$$
X=Y S(Y)=Y\left(1-\frac{(j-2)}{(j-1)} \epsilon(Y) \cos \omega t\right)^{1 /(2-j)}
$$

$$
\begin{aligned}
P_{X}= & {\left[P_{Y}-\frac{W^{\prime}(Y)}{T^{2}(Y)} \frac{\sin \omega t}{\omega}\right] T(Y) } \\
= & P_{Y}\left[1-\frac{(j-2)}{(j-1)} \epsilon(Y) \cos \omega t\right]^{(j-1) /(j-2)} \\
& -\frac{W^{\prime}(Y)}{\left[1-\frac{(j-2)}{(j-1)} \epsilon(Y) \cos \omega t\right]^{(j-1) /(j-2)} \frac{\sin \omega t}{\omega} .}
\end{aligned}
$$

From these expressions it can be seen that the first term in the generating function accounts for extraction of fast motion in the position coordinate, whereas the second term represents a gauge transformation chosen to give a conjugate momentum $P_{Y}$, which results in the simplest form of the associated Hamiltonian. This gauge transformation accounts for subtraction of fast motion in the momentum. [It should be noted that, depending on the time, the coordinate transformation (A4) does not cover the full $X$ or $Y$ axis. The maximum length of the missing interval is closely related to the value of the expansion parameter $\epsilon$.]

The second canonical transformation has the same structure as the first one, although it looks more complicated. This is due to the more-complicated analytical expression - in terms of $V$ and $W$-for the time-varying terms and to the presence of the spatially dependent mass correction in the intermediate Hamiltonian $H_{Y}$. We have

$$
\begin{aligned}
\bar{F}\left(P_{Y}, Z\right)= & -P_{Y} Z \bar{S}(Z) \\
& -\int^{Z} d z \frac{V_{\mathrm{Kap}}^{\prime}(z)}{[T(z \bar{S}(z)) \bar{T}(z)]^{2}} \\
& \times \frac{\left[4 \frac{V}{W} \sin \omega t-\frac{1}{2} \sin 2 \omega t\right]}{\omega},
\end{aligned}
$$

where

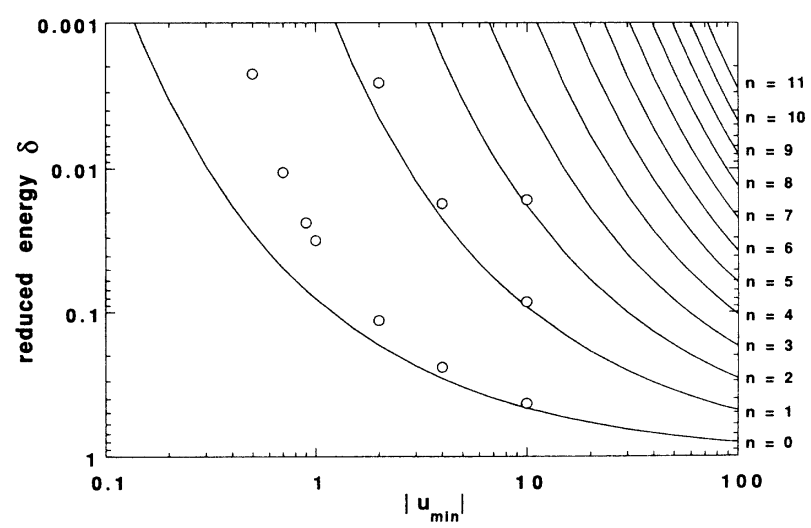

FIG. 6. Reduced eigenenergies $\delta$. Circles show numerical integration of the Schrödinger equation (34). Lines show exact WKB evaluation, which for the parameter range shown here cannot be distinguished from the predictions of Eq. (36). 


$$
\begin{aligned}
& \bar{S}(Z)=\left[1-\frac{(2 j-4)}{(2 j-3)} \frac{V_{\mathrm{Kap}}^{\prime \prime}(Z)}{M \omega^{2}}\left[4 \frac{V}{W} \cos \omega t-\frac{1}{4} \cos 2 \omega t\right]\right]^{1 /(4-2 j)}, \\
& \bar{T}(Z)=\left[1-\frac{(2 j-4)}{(2 j-3)} \frac{V_{\mathrm{Kap}}^{\prime \prime}(Z)}{M \omega^{2}}\left[4 \frac{V}{W} \cos \omega t-\frac{1}{4} \cos 2 \omega t\right]\right]^{(2 j-3) /(2 j-4)} .
\end{aligned}
$$

For the transformation relations between final and intermediate coordinates $Z$ and $Y$ and between momenta $P_{Z}$ and $P_{Y}$ we then obtain

$$
\begin{aligned}
Y= & Z \bar{S}(Z)=Z\left[1-\frac{(2 j-4)}{(2 j-3)} \frac{V_{\mathrm{Kap}}^{\prime \prime}(Z)}{M \omega^{2}}\left[4 \frac{V}{W} \cos \omega t-\frac{1}{4} \cos 2 \omega t\right]\right]^{1 /(4-2 j)}, \\
P_{Y}= & {\left[P_{Z}-\frac{V_{\mathrm{Kap}}^{\prime}(Z)}{[T(Z \bar{S}(Z)) \bar{T}(Z)]^{2}} \frac{\left(4 \frac{V}{W} \sin \omega t-\frac{1}{2} \sin 2 \omega t\right]}{\omega}\right] \bar{T}(Z) } \\
= & P_{Z}\left[1-\frac{(2 j-4)}{(2 j-3)} \frac{V_{\mathrm{Kap}}^{\prime \prime}(Z)}{M \omega^{2}}\left[4 \frac{V}{W} \cos \omega t-\frac{1}{4} \cos 2 \omega t\right]\right]^{(2 j-3) /(2 j-4)} \\
& -\left[1-\frac{(j-2)}{(j-1)} \cos \omega t \epsilon\left\{Z\left[1-\frac{(2 j-4) V_{\mathrm{Kap}}^{\prime \prime}(Z)}{(2 j-3) M \omega^{2}}\left[4 \frac{V}{W} \cos \omega t-\frac{1}{4} \cos 2 \omega t\right]\right]^{1 /(4-2 j)}\right\}\right]^{(2-2 j) /(j-2)} \\
& \times \frac{V_{\mathrm{Kap}}^{\prime}(Z)}{\left[1-\frac{(2 j-4)}{(2 j-3)} \frac{V_{\mathrm{Kap}}^{\prime \prime}(Z)}{M \omega^{2}}\left[4 \frac{V}{W} \cos \omega t-\frac{1}{4} \cos 2 \omega t\right]\right]^{(2 j-3) /(2 j-4)} \frac{\frac{1}{2} \sin 2 \omega t}{\omega} .}
\end{aligned}
$$

The final form for the classical Hamiltonian is

$$
\begin{array}{r}
H_{Z}=H_{Y}+\frac{\partial \bar{F}}{\partial t}=\frac{P_{Z}^{2}}{2 M}+V(Z)+V_{\mathrm{Kap}}(Z)+\epsilon(Z)\left[-\frac{P_{Z}^{2}}{M} \cos \omega t+V_{\mathrm{Kap}}(Z)\left[\cos \omega t \frac{4 j-5}{3 j-4}+\cos 3 \omega t \frac{1}{3 j-4}\right.\right. \\
\left.\left.-16 \frac{j-1}{3 j-4} \frac{V}{W} \cos 2 \omega t\right]\right]+\cdots .
\end{array}
$$

(A10)

We consider it remarkable that there are no time-independent corrections of first order to the Kapitza Hamiltonian which consists of the first three terms on the right-hand side of (A10).

\section{APPENDIX B: QUANTUM-MECHANICAL DESCRIPTION}

The unitary operator in Eq. (22) results in the position and momentum operator transformations

$$
\begin{aligned}
& X=Y S(Y) \\
& P_{X}=\frac{1}{2}\left[\left(P_{Y}-\frac{W^{\prime}(Y)}{T^{2}(Y)} \frac{\sin \omega t}{\omega}\right) T(Y)+T(Y)\left[P_{Y}-\frac{W^{\prime}(Y)}{T^{2}(Y)} \frac{\sin \omega t}{\omega}\right)\right] .
\end{aligned}
$$

The unitary operator corresponding to the second canonical transformation is given by

$$
\begin{aligned}
\bar{U}=\exp \left[-\frac{i}{\hbar} \int\right. & \left.d z \frac{V_{\mathrm{Kap}}^{\prime}(z)}{[T(z \bar{S}(z)) \bar{T}(z)]^{2}} \frac{4 \frac{V}{W} \sin \omega t-\frac{1}{2} \sin 2 \omega t}{\omega}\right) \\
& \times \exp \left[-\frac{i}{2 \hbar}\left[P_{Z} \frac{V_{\mathrm{Kap}}^{\prime}(Z)}{M \omega^{2}}+\frac{V_{\mathrm{Kap}}^{\prime}(Z)}{M \omega^{2}} P_{Z}\right]\left(4 \frac{V}{W} \cos \omega t-\frac{1}{4} \cos 2 \omega t\right]\right),
\end{aligned}
$$


which finally yields

$$
H_{Z}=H_{Y}+i \hbar \bar{U} \frac{\partial \bar{U}^{+}}{\partial t}=H_{\mathrm{Kap}}+\Delta H_{Z}
$$

with

$$
\begin{aligned}
\Delta H_{Z}= & -\frac{1}{4 M}\left[P_{Z}^{2} \epsilon(Z)+2 P_{Z} \epsilon(Z) P_{Z}+\epsilon(Z) P_{Z}^{2}\right] \cos \omega t \\
& +\epsilon(Z) V_{\mathrm{Kap}}(Z)\left(\cos \omega t \frac{4 j-5}{3 j-4}+\cos 3 \omega t \frac{1}{3 j-4}-16 \frac{j-1}{3 j-4} \frac{V}{W} \cos 2 \omega t\right)+\cdots .
\end{aligned}
$$

We end the quantum-mechanical description by considering the WKB approximation for Eq. (34) in more detail. We form the phase integral and set it equal to a quantized volume in phase space,

$$
\int_{\xi_{1}}^{\xi_{2}} d \xi\left[\delta+\frac{3}{2 \xi^{2}}-\frac{1}{2 \xi^{6}}\right]^{1 / 2}=\int_{y_{1}}^{y_{2}} d y \frac{\left(-y^{3}+\frac{3}{2} y^{2}-\frac{1}{2} \delta^{2}\right]^{1 / 2}}{2 y^{2}}=\frac{\left(n+\frac{1}{2}\right) \pi}{\sqrt{\left|u_{\min }\right|}} .
$$

The roots of the cubic function inside the square root are given by

$$
y_{0}=\frac{1}{2}+\cos \left(\frac{1}{3} \cos ^{-1}\left(1-2 \delta^{2}\right)+\frac{2 \pi}{3}\right), y_{1}=\frac{1}{2}+\cos \left[\frac{1}{3} \cos ^{-1}\left(1-2 \delta^{2}\right)+\frac{4 \pi}{3}\right], \quad y_{2}=\frac{1}{2}+\cos \left[\frac{1}{3} \cos { }^{-1}\left(1-2 \delta^{2}\right)\right],
$$

where the last two roots, $y_{1}$ and $y_{2}$, are the inner and outer classical turning points, respectively.

The phase integral can be evaluated exactly in terms of elliptic functions to give

$$
\frac{3}{2} \sqrt{y_{2}-y_{0}}\left(\frac{1-y_{0}}{y_{2}-y_{0}} K\left(\frac{y_{2}-y_{1}}{y_{2}-y_{0}}\right)-E\left(\frac{y_{2}-y_{1}}{y_{2}-y_{0}}\right)\right]=\frac{\left(n+\frac{1}{2}\right) \pi}{\sqrt{\left|u_{\min }\right|}} .
$$

Here the notation of [19] has been used.

The allowed energy values are obtained from this transcendental equation. Figure 6 shows plots of the energy eigenvalues for Eq. (34), obtained from numerical integration of this differential equation, numerical solution of (B7), and application of Eq. (36).

[1] S. Chu, J. E. Bjorkholm, A. Ashkin, and A. Cable, Phys. Rev. Lett. 57, 314 (1986).

[2] A. Aspect, J. Dalibard, A. Heidmann, C. Salomon, and C. Cohen-Tannoudjii, Phys. Rev. Lett. 57, 1688 (1986).

[3] E. L. Raab, M. Prentiss, A. Cable, S. Chu, and D. E. Pritchard, Phys. Rev. Lett. 59, 2631 (1987).

[4] W. D. Phillips, P. L. Gould, and P. D. Lett., Sicience 239, 877 (1988).

[5] P. D. Lett, R. N. Watt, C. I. Westbrook, W. D. Phillips, P. L. Gould, and H. J. Metcalf, Phys. Rev. Lett. 61, 169 (1988).

[6] R. J. Cook, D. G. Shankland, and A. L. Wells, Phys. Rev. A 31, 564 (1985).

[7] M. Combescure, Ann. Inst. Henri Poincaré 44, 293 (1986).

[8] L. S. Brown, Phys. Rev. Lett. 66, 527 (1991).

[9] W. Paul, Rev. Mod. Phys. 62, 531 (1990), and references cited therein.

[10] H. Lamb, Dynamics (Cambridge University, Cambridge, England, 1929), pp. 266-271.

[11] L. D. Landau and E. M. Lifshitz, Quantum Mechanics
(Pergamon, Oxford, 1977), pp. 54, 114-117.

[12] K. M. Case, Phys. Rev. 80, 797 (1950).

[13] P. M. Morse and H. Feshbach, Methods of Theoretical Physics (McGraw-Hill, New York, 1953), Vol. II, pp. 1665-1667.

[14] P. L. Kapitza, Zh. Eksp. Teor. Fiz. 21, 588 (1951). An English translation appears in Collected Papers of P. L. Kapit$z a$, edited by D. Ter Haar (Pergamon, Oxford, 1965), Vol. II.

[15] L. D. Landau and E. M. Lifshitz, Mechanics (Pergamon, Oxford, 1976), pp. 93-95.

[16] H. Goldstein, Classical Mechanics, 2nd ed. (AddisonWesley, Reading, MA, 1980).

[17] A. J. Lichtenberg and M. A. Lieberman, Regular and Stochastic Motion (Springer-Verlag, New York, 1983).

[18] J. Guckenheimer and P. Holmes, Nonlinear Oscillations, Dynamical Systems and Bifurcations of Vector Fields (Springer-Verlag, New York, 1983).

[19] Handbook of Mathematical Functions, edited by $\mathbf{M}$. Abramowitz and I. A. Stegun (Wiley, New York, 1972). 